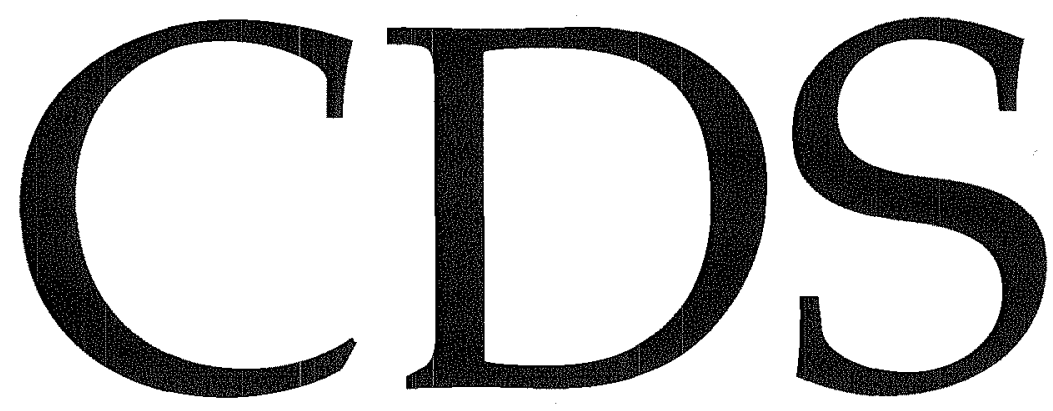

TECHNICAL MEMORANDUM NO. CIT-CDS 94-008

March 21, 1994

"Optimal and Robust Design of Integrated Control and Diagnostic Modules"

Matthew L. Tyler and Manfred Morari

Control and Dynamical Systems

California Institute of Technology

Pasadena, CA 91125 


\title{
Optimal and Robust Design of Integrated Control and Diagnostic Modules
}

\author{
Matthew L. Tyler * Manfred Morari ${ }^{\dagger}$ \\ Chemical Engineering, 210-41 \\ California Institute of Technology \\ Pasadena, CA 91125
}

\begin{abstract}
The problem of designing an integrated control and diagnostic module is considered. The four degree of freedom controller is recast into a general framework wherein results from optimal and robust control theory can be easily implemented. For the case of an $\mathcal{H}_{2}$ objective, it is shown that the optimal control-diagnostic module involves constructing an optimal controller, closing the loop with this controller, and then designing an optimal diagnostic module for the closed loop. When uncertain plants are involved, this two-step method does not lead to reasonable diagnostics, and the control and diagnostic modules must be synthesized simultaneously. An example shows how this design can be accomplished with available methods.
\end{abstract}

\section{Introduction}

The problem of designing filters for the monitoring and detection of system faults has received considerable attention since the seminal work of Beard [2] and Jones [9]. The survey paper [14] provides an early summary of work in this area, and [7] provides a more recent account. Although the majority of results for model based fault detection are based solely upon nominal models, a few significant studies which incorporate uncertain models have appeared $[8,10,3]$. However, the methods developed in these references are unable to incorporate the norm bounded uncertainty descriptions commonly employed in control synthesis. In addition, for feedback systems, these methods do not consider the interaction between detection and control, i.e. the detection algorithms consider the effect of known inputs on the outputs, but do not consider the effect of the output on the input.

Nett [11] introduced the four degree of freedom, or four parameter, controller which integrates control and diagnostics. By parameterizing all stabilizing controllers of this form, he was able to elucidate tradeoffs involving both controller performance and diagnostic performance. In addition to diagnostic tradeoffs such as between the detection of sensor and actuator faults, Nett et al. [12] have shown that when uncertain models are used, control performance must be traded off against

\footnotetext{
*Supported by Fannie and John Hertz Foundation.

${ }^{\dagger}$ Author to whom all correspondence should be addressed: phone (818)395-4186, fax (818)568-8743, e-mail MM@IMC.CALTECH.EDU.
} 
diagnostic performance. This work suggests that both control and diagnostic modules must be designed together.

Although several years have passed since Nett parameterized the four degree of freedom controller, it has not found widespread use due to the fact that a systematic synthesis method which guarantees robust control and diagnostic performance in the face of uncertainty does not exist. Notable attempts at outlining synthesis methods can be found in [12] and [6]. The former proposes a four step procedure in which each degree of freedom is considered successively. The latter employs a method which first designs a nominal control module with robust stability in an $l_{\infty}$ framework, and then designs the diagnostic module on the resultant closed loop.

In this paper, we show how the four degree of freedom controller is simply a special case of the general interconnection structure used in modern control synthesis methods. Viewed in this framework, we employ results from $\mathcal{H}_{2^{-}}, \mathcal{H}_{\infty^{-}}$, and $\mu$-synthesis methods to the design of integrated control and diagnostic systems. Through an example, we show that for uncertain systems, robust performance of both control and diagnostics requires that these two modules be designed simultaneously.

\section{Four Degree of Freedom Controller}

Consider a general multi-input, multi-output (MIMO) system $G$. Partition the inputs to $G$ into uncontrolled inputs $d$, and controlled inputs $u$. The signal $d$ represents noises, disturbances, and faults. Also partition the outputs as those outputs used by the control algorithm $(y)$ from those not used $(z)$, yielding the following partition for $G$ :

$$
\left[\begin{array}{l}
z \\
y
\end{array}\right]=\left[\begin{array}{ll}
G_{11} & G_{12} \\
G_{21} & G_{22}
\end{array}\right]\left[\begin{array}{l}
d \\
u
\end{array}\right] .
$$

The four degree of freedom controller has access to a reference signal $r$ as well as the measurement $y$, and returns not only the control moves $u$, but also a diagnostic alarm signal $a$ :

$$
\left[\begin{array}{l}
a \\
u
\end{array}\right]=\left[\begin{array}{ll}
K_{11} & K_{12} \\
K_{21} & K_{22}
\end{array}\right]\left[\begin{array}{l}
r \\
y
\end{array}\right] .
$$

Let the input $d$ be described by $d=\left[f^{T}, n^{T}\right]^{T}$, where $f$ represents faults, and $n$ represents noises and disturbances which occur under normal operation. Nett et al. [12] have elucidated several algebraic tradeoffs involved with fault detection which we will not repeat, except to comment that in general, one must tradeoff the ability to detect input or actuator faults from output or sensor faults.

Of more interest for our discussion is the tradeoff between diagnostics and control. For example, consider the block diagram shown in Figure 1. Suppose we desire that the alarm signal $a$ tracks input faults $f_{i}$. The alarm signal $a$ is given by

$$
a=T_{a r} r+T_{a f_{\circ}}\left(f_{\circ}+n_{\circ}+G_{22}\left(f_{i}+n_{i}\right)\right)
$$

where the perturbed transfer functions $T_{a r}$ and $T_{a f_{o}}$ are given by

$$
T_{a r}=K_{11}+K_{12}\left(I-S_{o} G_{22} \Delta_{u} W_{u} K_{22}\right)^{-1} S_{o}\left(G_{22}+G_{22} \Delta_{u} W_{u}\right) K_{21},
$$




$$
T_{a f_{o}}=\left(I-S_{o} G_{22} \Delta_{u} W_{u} K_{22}\right)^{-1} S_{o}
$$

and $S_{o}=\left(I-G K_{22}\right)^{-1}$ is the output sensitivity function. For nominal systems, $K$ can easily be designed so that $T_{a r}=0$ [11]; however, as the above expression for this transfer function suggests, model uncertainty prohibits such a design, and the objective of making $T_{a r}$ robustly small will compete with disturbance rejection and reference tracking objectives.

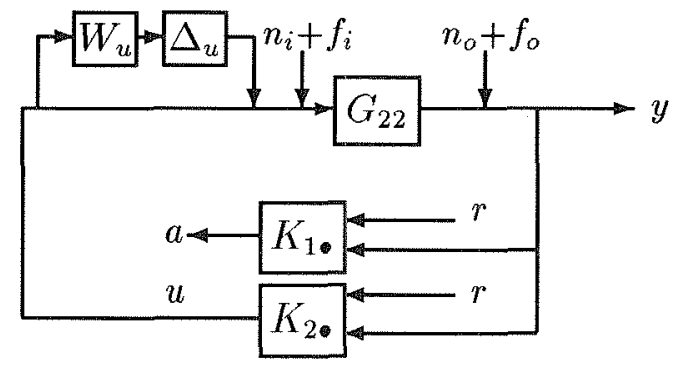

Figure 1: Control and diagnostic configuration for uncertain system.

\section{General Framework}
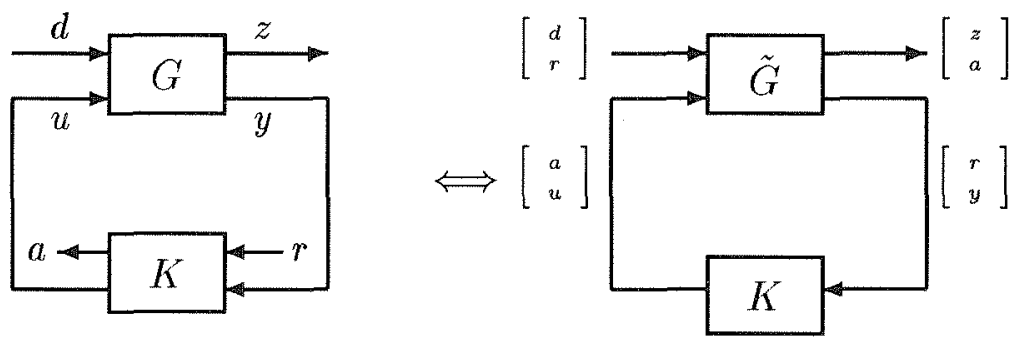

Figure 2: Equivalent four parameter controller configurations.

Consider the plant and four parameter controller as in (1) and (2). As written, the reference signal $r$ is not an input to $G$, and the output alarm $a$ is not an output of $G$. We would like to obtain a reconfigured system $\tilde{G}$ so that all the inputs are inputs to $\tilde{G}$, all the outputs are outputs of $\tilde{G}$, and the closed loop systems are equivalent, as shown in Figure 2. This can easily be accomplished with the following augmented plant:

$$
\tilde{G}=\left[\begin{array}{cccc}
G_{11} & 0 & 0 & G_{12} \\
0 & 0 & I & 0 \\
0 & I & 0 & 0 \\
G_{21} & 0 & 0 & G_{22}
\end{array}\right]
$$

We may therefore view the four degree of freedom controller as a special case of the more general interconnection input-output system. Several advantages arise by viewing the control-diagnostic system in this light. First, the parameterization of stabilizing controllers can be viewed as a simple application of the Youla parameterization, as shown in Section 4. Second, optimal control results, 
such as those found in [5], may be applied in a straightforward way. Finally, robust analysis and synthesis of the control-diagnostic module may be carried out in a systematic fashion using readily available tools such as those available in [1].

\section{Reconciliation between Four-Parameter Parameterization and Youla Parameterization}

By using the augmented $\tilde{G}$, the parameterization of stabilizing controllers is a straightforward application of the Youla parameterization. In order to analyze stability, we need only consider the bottom right partition of $\tilde{G}$,

$$
\tilde{G}_{22}=\left[\begin{array}{cc}
0 & 0 \\
0 & G_{22}
\end{array}\right] \text {. }
$$

Suppose a doubly coprime factorization of $G_{22}$ is given by

$$
G_{22}=N_{G} D_{G}^{-1}=\tilde{D}_{G}^{-1} \tilde{N}_{G}
$$

where

$$
\left[\begin{array}{cc}
V_{G} & U_{G} \\
-\tilde{N}_{G} & \tilde{D}_{G}
\end{array}\right]=\left[\begin{array}{cc}
D_{G} & -\tilde{U}_{G} \\
N_{G} & \tilde{V}_{G}
\end{array}\right]^{-1}
$$

Then a doubly coprime factorization of $\tilde{G}_{22}$ is given by

$$
\begin{aligned}
& M_{\tilde{G}}=\left[\begin{array}{cc}
0 & 0 \\
0 & M_{G}
\end{array}\right], M \in\{N, U, \tilde{N}, \tilde{U}\}, \\
& M_{\tilde{G}}=\left[\begin{array}{cc}
I & 0 \\
0 & M_{G}
\end{array}\right], M \in\{D, V, \tilde{D}, \tilde{V}\} .
\end{aligned}
$$

The parameterization of all stabilizing controllers can be found in [13] and is given by:

$$
K=\left(\tilde{V}_{\tilde{G}}+Q \tilde{N}_{\tilde{G}}\right)^{-1}\left(-\tilde{U}_{\tilde{G}}+Q \tilde{D}_{\tilde{G}}\right)
$$

where $Q$ is any stable transfer function. By partitioning $Q$ and substituting the coprime factorization of $\tilde{G}_{22}$ given in (7) into (8), $K$ can be parameterized in terms of the factorization of $G_{22}$, resulting in

$$
K=\left[\begin{array}{ll}
K_{11} & K_{12} \\
K_{21} & K_{22}
\end{array}\right]
$$

where

$$
\begin{aligned}
& K_{11}=Q_{11}-Q_{12} \tilde{N}_{G}\left(V_{G}+Q_{22} \tilde{N}_{G}\right)^{-1} Q_{21} \\
& K_{12}=Q_{12} \tilde{D}_{G}-Q_{12} \tilde{N}_{G}\left(V_{G}+Q_{22} \tilde{N}_{G}\right)^{-1}\left(Q_{22} \tilde{D}_{G}-U_{G}\right) \\
& K_{21}=\left(V_{G}+Q_{22} \tilde{N}_{G}\right)^{-1} Q_{21} \\
& K_{22}=\left(V_{G}+Q_{22} \tilde{N}_{G}\right)^{-1}\left(Q_{22} \tilde{D}_{G}-U_{G}\right)
\end{aligned}
$$


Using algebraic manipulations and properties of the doubly coprime factorization, one can show

$$
K_{12}=Q_{12}\left(N_{G} Q_{22}+\tilde{V}_{G}\right)^{-1}
$$

in which case $K$ has the same form as in [11].

\section{5. $\mathcal{H}_{2}$ Optimal Control and Diagnostics}

In this section, we consider the problem of designing an $\mathcal{H}_{2}$ optimal controller which provides a nominally small output and also tracks faults. Let $d=\left[f^{T}, n^{T}, r^{T}\right]^{T}$, and $y=\left[\hat{y}^{T}, r^{T}\right]^{T}$. Let the transfer function which maps $\left[d^{T}, u^{T}\right]$ onto $\left[z^{T}, y^{T}\right]$ be given by:

$$
G_{c}=\left[\begin{array}{c|cc}
A_{c} & B_{c 1} & B_{c 2} \\
\hline C_{c 1} & 0 & D_{c 12} \\
C_{c 2} & D_{c 21} & 0
\end{array}\right]
$$

The following assumptions simplify the analysis, and relaxing these assumptions do not change the nature of the results:

1. $D_{\mathrm{c} 12}^{*} C_{\mathrm{c} 1}=0$.

2. $D_{c 21} D_{c 21}^{*}=I, D_{c 12}^{*} D_{c 12}=I$.

Assumption 1 implies that the objective function has no cross terms of the form $\langle x, u\rangle$, where $x$ represents the system states. Assumption 2 can easily be relaxed by introducing scaling matrices. Note that the common assumption $B_{c 1} D_{c 21}^{*}=0$ is not meaningful since the signal $r$ is directly measured.

A general diagnostic objective function is taken as the output of the following transfer function which takes $d$ and $a$ to $z_{d}$ :

$$
G_{d}=\left[\begin{array}{c|cc}
A_{d} & B_{d 1} & B_{d 2} \\
\hline C_{d 1} & 0 & D_{d 12}
\end{array}\right] .
$$

For example, if the alarm signal should track actuator faults, then an appropriate choice of the signal $z_{d}$ may be $\left[\begin{array}{c}W_{d}\left(a-f_{i}\right) \\ W_{a}(a)\end{array}\right]$. By combining the two systems to obtain the objective output $\left[z^{T}, z_{d}^{T}\right]^{T}$, the following interconnection structure is obtained:

$$
\tilde{G}=\left[\begin{array}{c|cc}
\tilde{A} & \tilde{B}_{1} & \tilde{B}_{2} \\
\hline \tilde{C}_{1} & 0 & \tilde{D}_{12} \\
\tilde{C}_{2} & \tilde{D}_{21} & 0
\end{array}\right],
$$

where the inputs are partitioned such that $\tilde{B}_{1}$ multiplies $\left[f^{T}, n^{T}, r^{T}\right]^{T}$ and $\tilde{B}_{2}$ multiplies $\left[a^{T}, u^{T}\right]^{T}$. The system matrices for $\tilde{G}$ are given by:

$$
\tilde{A}=\left[\begin{array}{cc}
A_{c} & 0 \\
0 & A_{d}
\end{array}\right], \tilde{B}_{1}=\left[\begin{array}{c}
B_{c 1} \\
B_{d 1}
\end{array}\right], \tilde{B}_{2}=\left[\begin{array}{cc}
0 & B_{c 2} \\
B_{d 2} & 0
\end{array}\right],
$$




$$
\begin{gathered}
\tilde{C}_{1}=\left[\begin{array}{cc}
C_{c 1} & 0 \\
0 & C_{d 1}
\end{array}\right], \tilde{D}_{12}=\left[\begin{array}{cc}
0 & D_{c 12} \\
D_{d 12} & 0
\end{array}\right], \\
\tilde{C}_{2}=\left[\begin{array}{ll}
C_{c 2} & 0
\end{array}\right], \tilde{D}_{21}=D_{c 21} .
\end{gathered}
$$

The $\mathcal{H}_{2}$ optimal controller can be found in [5] and is given by

$$
K=\left[\begin{array}{c|c}
\tilde{A}+\tilde{B}_{2} F_{2}+L_{2} \tilde{C}_{2} & -L_{2} \\
\hline F_{2} & 0
\end{array}\right],
$$

where

$$
-L_{2}=Y \tilde{C}_{2}^{*}+\tilde{B}_{1} \tilde{D}_{21}^{*},-F_{2}=\tilde{B}_{2}^{*} X
$$

and $X$ and $Y$ are the solutions to the algebraic Riccati equations determined respectively by the Hamiltonian matrices $H_{2}$ and $J_{2}$ :

$$
\begin{gathered}
H_{2}=\left[\begin{array}{cc}
\tilde{A} & -\tilde{B}_{2} \tilde{B}_{2}{ }^{*} \\
-\tilde{C}_{1}{ }^{*} \tilde{C}_{1} & -\tilde{A}^{*}
\end{array}\right], \\
J_{2}=\left[\begin{array}{cc}
\tilde{A}^{*}-\tilde{C}_{2}^{*} \tilde{D}_{21} \tilde{B}_{1}^{*} & -\tilde{C}_{2}^{*} \tilde{C}_{2} \\
-\tilde{B}_{1}\left(I-\tilde{D}_{21}^{*} \tilde{D}_{21}\right) \tilde{B}_{1}{ }^{*} & -\tilde{A}+\tilde{B}_{1} \tilde{D}_{21}^{*} \tilde{C}_{2}
\end{array}\right] .
\end{gathered}
$$

By partitioning $X$ and $Y$ in the obvious fashion and introducing the following matrices:

$$
\begin{aligned}
& M=\tilde{A}-\tilde{B}_{1} \tilde{D}_{21}^{*} \tilde{C}_{2}=\left[\begin{array}{cc}
A_{c}-B_{c 1} D_{c 21}^{*} C_{c 2} & 0 \\
-B_{d 1} D_{c 21}^{*} C_{c 2} & A_{d}
\end{array}\right] \\
& R=\tilde{B}_{1}\left(I-\tilde{D}_{21}^{*} \tilde{D}_{21}\right) \tilde{B}_{1}^{*}=\left[\begin{array}{cc}
B_{c 1}\left(I-D_{c 21}^{*} D_{c 21}\right) B_{c 1}^{*} & B_{c 1}\left(I-D_{c 21}^{*} D_{c 21}\right) B_{d 1}^{*} \\
B_{d 1}\left(I-D_{c 21}^{*} D_{c 21}\right) B_{c 1}^{*} & B_{d 1}\left(I-D_{c 21}^{*} D_{c 21}\right) B_{d 1}^{*}
\end{array}\right],
\end{aligned}
$$

the two Riccati equations can be written as the following coupled matrix equations:

$$
\begin{gathered}
A_{c}^{*} X_{11}+X_{11} A_{c}-X_{11} B_{c 2} B_{c 2}^{*} X_{11}+C_{c 1}^{*} C_{c 1}-X_{12} B_{d 2} B_{d 2}^{*} X_{12}^{*}=0 \\
A_{d}^{*} X_{22}+X_{22} A_{d}+C_{d 1}^{*} C_{d 1}-X_{22} B_{d 2} B_{d 2}^{*} X_{22}-X_{12} B_{c 2} B_{c 2}^{*} X_{12}^{*}=0, \\
\left(A_{c}^{*}-X_{11} B_{c 2} B_{c 2}^{*}\right) X_{12}+X_{12}\left(A_{d}-B_{d 2} B_{d 2}^{*} X_{22}\right)=0 \\
M_{11} Y_{11}+Y_{11} M_{11}^{*}-Y_{11} C_{c 2}^{*} C_{c 2} Y_{11}+R_{11}=0, \\
M_{11} Y_{12}+Y_{12} M_{22}^{*}+Y_{11} M_{21}^{*}-Y_{11} C_{c 2}^{*} C_{c 2} Y_{12}+R_{12}=0 \\
M_{22} Y_{22}+M_{21} Y_{12}+Y_{22} M_{22}^{*}+Y_{12}^{*} M_{21}^{*}+R_{22}\left(-Y_{12}^{*} C_{c 2}^{*} C_{c 2} Y_{12}=0\right.
\end{gathered}
$$

We see that the equations for $Y$ are coupled only in one direction, i.e. $Y_{12}$ depends upon $Y_{11}$, but $Y_{11}$ can be found independently of $Y_{12}$. In fact, none of the parameters for $Y_{11}$ depend upon the diagnostic terms, so $Y_{11}$ is the same solution that would be obtained if diagnostics were not 
considered. As for the $X$ equations, the second is linear and homogeneous in $X_{12}$, so $X_{12}=0$. This decouples the equations. $F_{2}$ and $L_{2}$ are then given by

$$
\begin{aligned}
& F_{2}=-\left[\begin{array}{cc}
B_{c 2}^{*} X_{11} & 0 \\
0 & B_{d 2}^{*} X_{22}
\end{array}\right], \\
& L_{2}=-\left[\begin{array}{c}
Y_{11} C_{c 2}^{*}+B_{c 1} D_{c 21}^{*} \\
Y_{12}^{*} C_{c 2}^{*}+B_{d 1} D_{c 21}^{*}
\end{array}\right] .
\end{aligned}
$$

Thus, for systems whose interconnection structure can be described by (13), the optimal control module is independent of the optimal diagnostic module for the $\mathcal{H}_{2}$ optimal solution. Thus, when considering only a nominal model, the two step procedure of designing an optimal control module, calculating the closed loop system, and then designing the optimal diagnostic module is equivalent to designing the optimal control and diagnostic module simultaneously. However, when uncertainty is considered, we will show by example that the two modules should be considered simultaneously.

The key feature of the system (13) which allows for the control to be designed independently of the diagnostic is that the alarm signal $a$ does not affect the states associated with $G_{c}$. This restriction is reasonable as its violation would imply that the overall objective included some crossterms between the output and the alarm. For example, in a stochastic setting, assuming the form (13) would allow objective function terms of the form $E\left(y^{T} y\right)$ and $E\left((a-f)^{T}(a-f)\right)$, but not $E\left(y^{T}(a-f)\right)$, where $E$ is the expectation operator.

\section{Robust Synthesis}

Viewing the integrated control-diagnostic problem as a simple case of the general interconnection framework as shown in Figure 2 allows the application of robust synthesis tools such as those in [1] in a straightforward fashion. In particular, a control-diagnostic module which guarantees robust performance may be synthesized using a two block $\mu$ structure, and the DK iteration method.

Consider a system with the structure shown in Figure 1. We would like to design a controldiagnostic module with the following properties:

1. The output signal tracks reference commands and is insensitive to actuator faults.

2. The alarm signal is large only when a fault has occurred.

3. Properties 1 and 2 hold in the presence of a bounded uncertainty.

Let $T_{c}$ be the transfer function from $\left[f^{T}, n^{T}, r^{T}\right]^{T}$ to $y$ - $r, T_{d}$ be the transfer function from $\left[f^{T}, n^{T}, r^{T}\right]^{T}$ to $a-f$, and $T_{a}$ be the transfer function from $\left[f^{T}, n^{T}, r^{T}\right]^{T}$ to $a$. A mathematical statement of the design objective is formulated as follows: Find a stabilizing $K$ such that

$$
\left\|\begin{array}{l}
W_{c} T_{c} \\
W_{d} T_{d} \\
W_{a} T_{a}
\end{array}\right\|_{\mathcal{H}_{\infty}} \leq 1 \text { for all } \Delta_{u} \mid\left\|\Delta_{u}\right\|_{\mathcal{H}_{\infty}} \leq 1 .
$$


Including a weight on $T_{a}$ is needed for the problem to be well posed. By introducing a full performance block $\Delta_{p}$, this problem can be stated equivalently as: Find a stabilizing $K$ such that

$$
\mu_{\left[\Delta_{p}\right.}{ }^{\Delta_{u}}\left(F_{l}(\tilde{G}, K)\right)<1,
$$

where $\tilde{G}$ is an appropriate interconnection structure which contains the weighting functions, and $F_{l}$ represents the lower linear fractional transformation.

Now let us consider as a specific example a second order, two-input, two-output system with actuator faults and measurement noise, i.e. $f=f_{i}, n=n_{0}$, and $f_{0}=n_{i}=0$. The frequency response for the nominal model is shown in Figure 3, and the system matrices are given by

$$
\begin{gathered}
A=\left[\begin{array}{cc}
-0.1536 & 0.1914 \\
-0.1583 & -0.5311
\end{array}\right], \\
B=\left[\begin{array}{cc}
-0.2290 & 0.1701 \\
-0.1719 & -0.0166
\end{array}\right], \\
C=\left[\begin{array}{cc}
0.1301 & -0.0210 \\
0.2538 & -0.1714
\end{array}\right], \\
D=0 .
\end{gathered}
$$

We assume an uncertainty of $10 \%$ in the gain of each input channel, and cover this model set by an input uncertainty, with $W_{u}=0.1 I, \Delta_{u}=\operatorname{diag}\left(\Delta_{1}, \Delta_{2}\right)$, where $\Delta_{1}$ and $\Delta_{2}$ depict $1 \times 1$ blocks, and $\left\|\Delta_{u}\right\|_{\mathcal{H}_{\infty}} \leq 1$. The performance weights $W_{c}, W_{d}$, and $W_{a}$ are diagonal, with the same weight on each component, and the magnitudes of these weights are shown in Figure 4. Note that the performance objective is not very aggressive, namely, we desire steady state reference tracking within $10 \%$. One would expect that this type of objective could be easily achieved.

Two controllers were synthesized. For the first controller, the DK iteration method was used to simultaneously design the control and diagnostic modules, and a final value of $\mu=0.95$ was achieved. We will refer to this controller as the one-step controller. In the second case, a robust control module was designed without considering the diagnostic objective. The resulting controller was used to close the loop. Synthesis of an $\mathcal{H}_{\infty}$ optimal diagnostic led to a value of $\mu \approx 12$. Several iterations on the DK scheme resulted in $\mu \approx 6.5$. We describe the control-diagnostic module which achieves the latter $\mu$ value as the two-step controller.

For both the one-step and the two-step control-diagnostic modules, the output was simulated with $\Delta_{u}=\operatorname{diag}(0.1,-0.1)$. The input consisted of a ramp actuator fault in the first channel which rose from 0 to 1 between time 100 and 200, and a set point step change of -1 for the second output at time 10, as shown in Figure 5. The results of the simulation are shown in Figure 6, which displays the plant output, and Figure 7 , which shows the filtered alarm signal $W_{d} a$.

From the simulations, one can make the following observations. The two-step control-diagnostic module provides much better performance in regard to reference tracking; however, the diagnostics are inadequate. A large false alarm occurs at time 10 when the reference signal changes. The one-step module does not issue a false alarm, but experiences significantly deteriorated reference tracking performance. This suggests that robust control-diagnostic performance requires detuning 

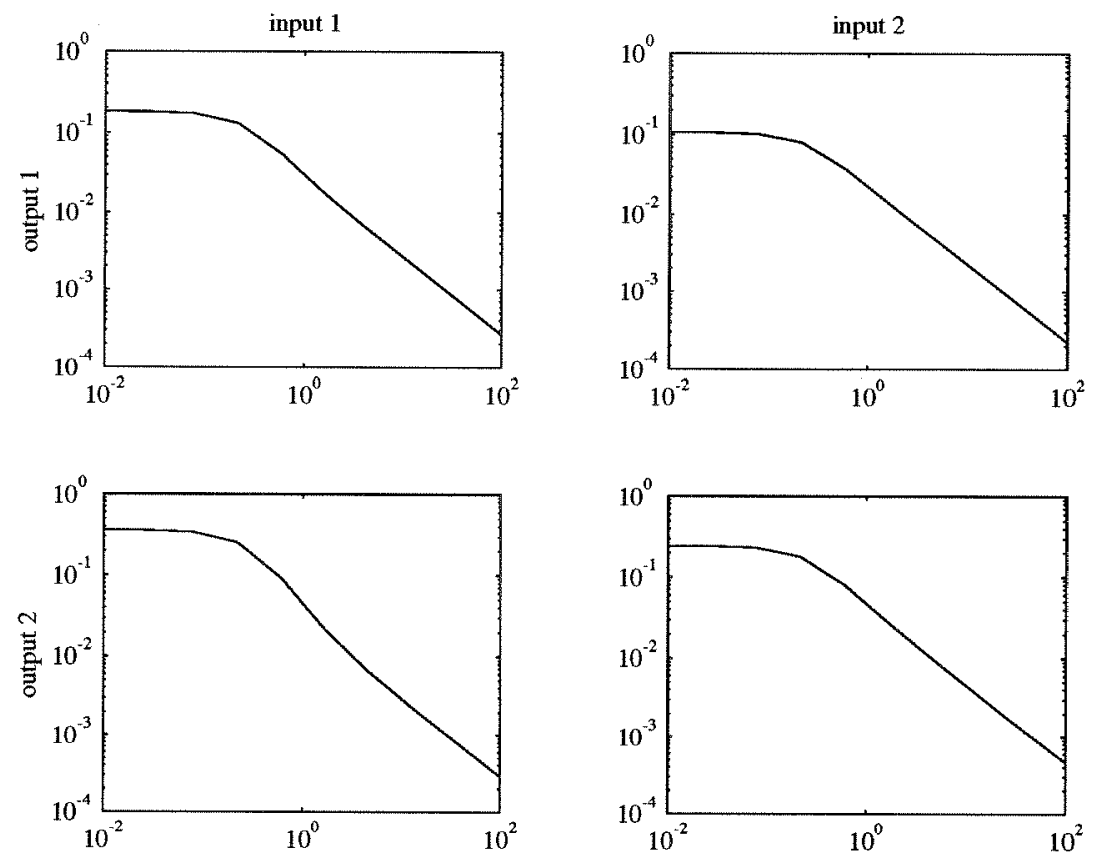

Figure 3: Frequency Response for plant.

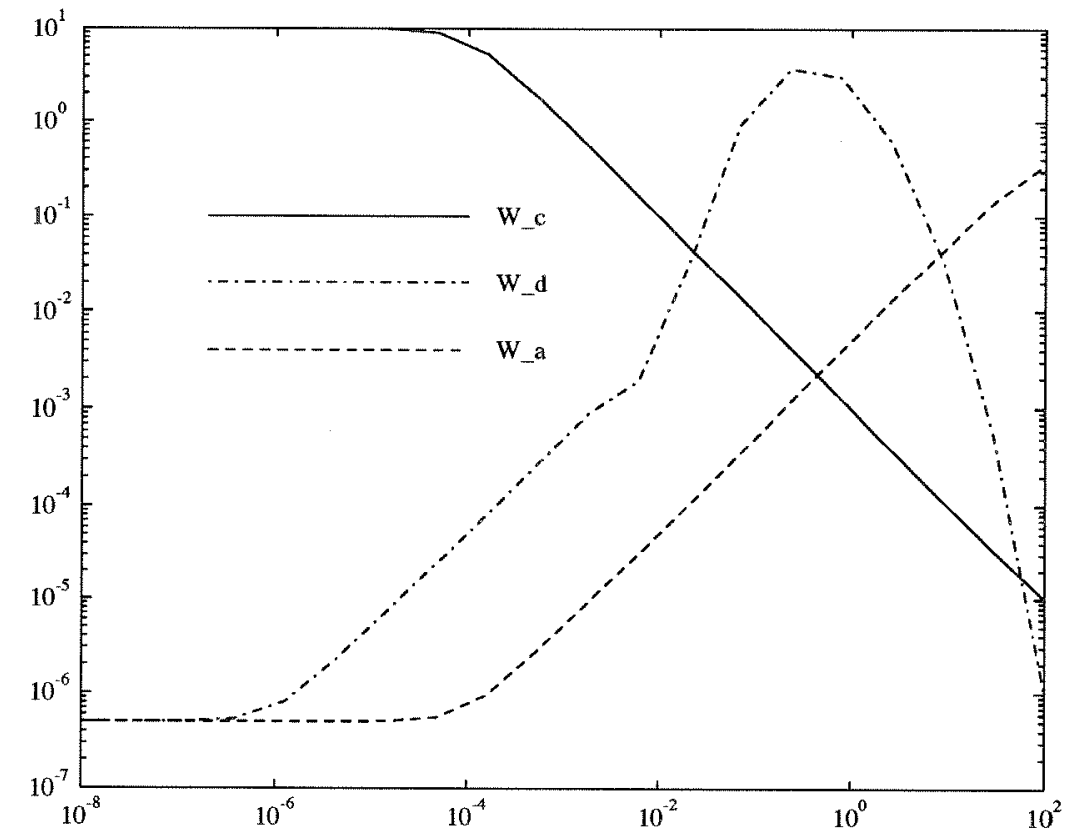

Figure 4: Performance Weights. 


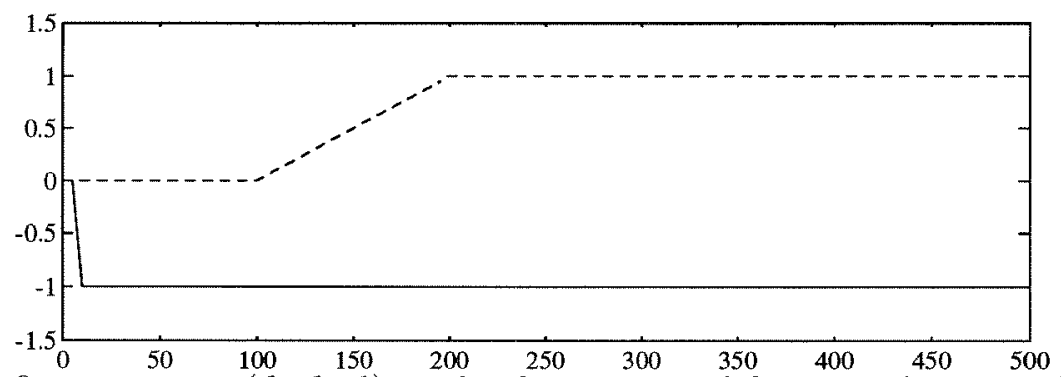

Figure 5: Fault in first actuator (dashed), and reference signal for second output (solid). All other inputs signals are zero.

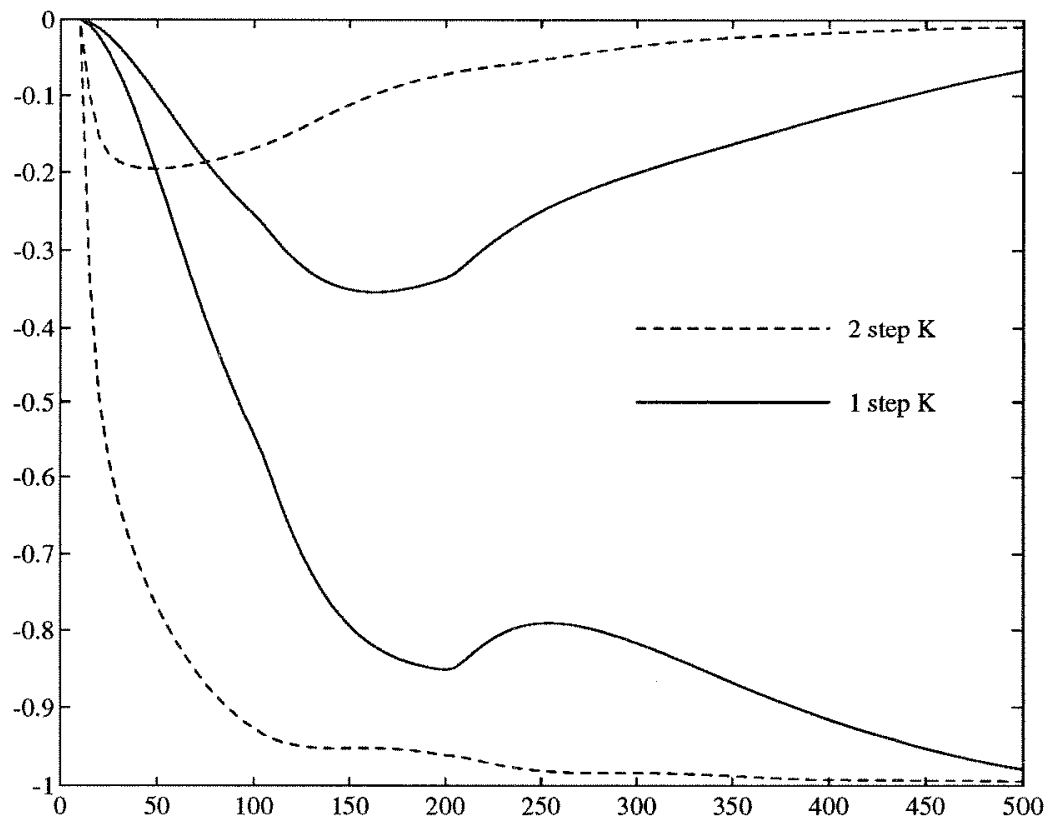

Figure 6: Plant Output for one-step and two-step controller designs.

of the control action to a much greater extent than would be expected when diagnostics are not considered.

For the one-step system, the alarm signal is raised when the slope of the fault changes, but not during the constant slope section of the fault ramp. This is due to the diagnostic weighting function $W_{d}$ which annihilates low frequency behavior. For this example, it was not possible to achieve robust performance with a weighting with significant low frequency energy. Roughly speaking, the facts that a signal $W_{u} \Delta_{u} u$ enters the system in the same way as the fault $f$ and that in steady state $u$ must change to track $r$ preclude the possibility of robust control and detection in the low frequency range.

The two-step controller achieves robust control performance, with $\mu \approx 0.1$ for the first step of this synthesis; however, the resulting control action leads to poor diagnostics. This suggests that methods for optimal or robust diagnostics which do not explicitly consider control action, such as in [4], will not work well for uncertain plants. 

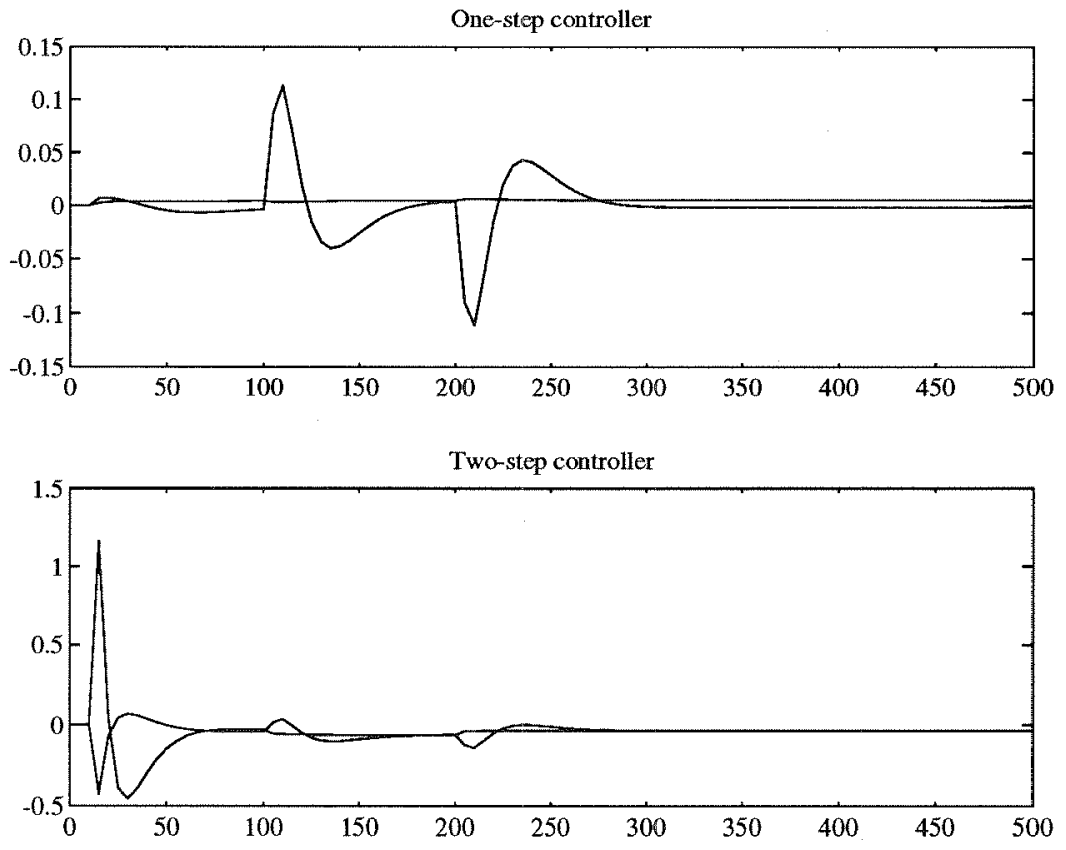

Figure 7: Filtered alarm signal $W_{d} a$ for one-step and two-step controller designs.

\section{Conclusions}

In this paper, we have shown how systems which integrate diagnostics and control can be designed using standard methods. For nominal models, $\mathcal{H}_{2}$ theory justifies designing the control and diagnostic modules successively; however, uncertainty requires that the design be simultaneous since diagnostic objectives may limit achievable performance.

An important question which we did not address in this study is the selection of performance measures for diagnostics. We considered the $\mathcal{H}_{2}$ norm for the nominal case, and the $\mathcal{H}_{\infty}$ norm for the robust case primarily due to the availability of methods to address these problems. In choosing a performance measure for a diagnostic system, one should consider the detection algorithm which will be used to determine when faults have occurred. 


\section{References}

[1] G. J. Balas, J. C. Doyle, K. Glover, A. K. Packard, and R. S. R. Smith. $\mu$-Analysis and Synthesis Toolbox ( $\mu$-Tools) : Matlab Functions for the Analysis and Design of Robust Control Systems. The Mathworks, Inc., Natick, MA, 1991. Computer Software.

[2] R. V. Beard. Failure Accommodation in Linear Systems through Self-reorganization. PhD thesis, MIT, Cambridge, MA, 1971.

[3] E. Y. Chow and A. S. Willsky. Analytical redundancy and the design of robust failure detection systems. IEEE Transactions on Automatic Control, 29:603-614, July 1984.

[4] X. Ding and P. M. Frank. Fault identification filter design via $\mathcal{H}_{\infty}$-optimization techniques. In Proc. IFAC Symp. on Id. B3 Sys. Param. Est., pages 219-224, Budapest, 1991.

[5] J. C. Doyle, K. Glover, P. Khargonekar, and B. Francis. State-space solutions to standard $\mathrm{H}_{2}$ and $H_{\infty}$ control problems. IEEE Transactions on Automatic Control, 34(8):831-847, Aug 1989.

[6] A. Elias-Juarez, A. Ajbar, and J. C. Kantor. Multiobjective $l_{\infty}$ design with integrated diagnostics. In Proceedings of the 1991 American Control Conference, pages 1671-1672, 1991.

[7] P. M. Frank. Fault diagnosis in dynamic systems using analytical and knowledge-based redundancy - a survey and some new results. Automatica, 26(3):459-474, 1990.

[8] P. M. Frank and J. Wünnenberg. Robust fault diagnosis using unknown input observer schemes. In R. Patton, P. Frank, and R. Clark, editors, Fault Diagnosis in Dynamic Systems, Theory and Applications, New York, New York, 1989. Prentice Hall.

[9] H. L. Jones. Failure Detection in Linear Systems. PhD thesis, MIT, Cambridge, MA, 1973.

[10] X. C. Lou, A. S. Willsky, and G. C. Verghese. Optimally robust redundancy relations for failure detection in uncertain systems. Automatica, 22(3):333-344, 1986.

[11] C. N. Nett. Algebraic aspects of linear control system stability. IEEE Transactions on Automatic Control, 31:941-949, 1986.

[12] C. N. Nett, C. A. Jacobson, and A. T. Miller. An integrated approach to controls and diagnostics: The 4-parameter controller. In Proceedings of the 1988 American Control Conference, pages $824-835,1988$.

[13] M. Vidyasagar. Control System Synthesis: A Factorization Approach. MIT Press, Cambridge, Massachusetts, 1985.

[14] A. S. Willsky. A survey of design methods for failure management in dynamical systems. Automatica, 12:601-611, 1976. 